\title{
El reciclaje y la expresión plástica en niños de inicial
}

\author{
Tatiana Aranda Vargas \\ tarandav@ucvvirtual.edu.pe \\ ORCID: 0000-0002-1324-1888 \\ Universidad Cesar Vallejo - Perú \\ Groberti Alfredo Medina Corcuera \\ grome@ucvvirtual.edu.pe \\ ORCID: 0000-0003-4035-157X \\ Universidad Cesar Vallejo - Perú
}

\section{RESUMEN}

El presente estudio tuvo como objetivo demostrar que la aplicación del programa de reciclaje a través de sesiones de clase y aprendizaje mejora la expresión plástica en niños de 4 años de la I.E. $\mathrm{N}^{\circ}$ 1678. El enfoque de estudio es cuantitativo, descriptivo - pre experimental aplicado en un grupo de niños tanto en el pre y post test. El marco poblacional estuvo compuesto de 189 niños de la I.E Rayitos de Luz, del distrito de Nuevo Chimbote, en las aulas de 3, 4 y 5 años. Asimismo, para el acopio de información se empleó una ficha de observación cuyo contenido tuvo 19 ítems. La hipótesis comprobó la incidencia de la aplicación del Programa de Reciclaje en la mejora significativa de la expresión plástica en los niños de 04 años que participaron en la evaluación. Concluyéndose, al analizar la prueba de T Student el hallazgo de diferencias significativas positivas entre la prueba tomada en el pre test y el pos test, estableciéndose un puntaje reflejado en la media de 32.27 y una desviación estándar (DE) de 10.43. Finalmente, se demostró que el programa de reciclaje mejora significativa la expresión plástica en los niños de 04 años en la I.E. $N^{\circ} 1678-2021$.

Palabras clave: reciclaje; expresión plástica; procedimental; conceptual; actitudinal. 


\title{
Recycling and plastic expression in kindergarten children
}

\begin{abstract}
The present study aimed to demonstrate that the application of the recycling program through class and learning sessions improves plastic expression in 4-year-old children of the I.E. $N^{\circ}$ 1678. The study approach is quantitative, descriptive - pre-experimental applied to a group of children both in the pre and post test. The population frame was made up of 189 children from the Rayitos de Luz I.E., from the Nuevo Chimbote district, in the 3, 4 and 5-year-old classrooms. Likewise, for the collection of information an observation card was used whose content had 19 items. The hypothesis verified the incidence of the application of the Recycling Program in the significant improvement of the plastic expression in the 04-year-old children who participated in the evaluation. Concluding, when analyzing the $\mathrm{T}$ Student test, the finding of significant positive differences between the test taken in the pre-test and the post-test, establishing a score reflected in the mean of 32.27 and a standard deviation (SD) of 10.43. Finally, it was shown that the recycling program significantly improves plastic expression in 04-yearold children in the I.E. $\mathrm{N}^{\circ} 1678$ - 2021.
\end{abstract}

Keywords: recycling; plastic expression; procedural; conceptual; attitudinal.

Artículo recibido: 15 noviembre. 2021 Aceptado para publicación: 10 diciembre 2021 Correspondencia: tarandav@ucvvirtual.edu.pe Conflictos de Interés: Ninguna que declarar 


\section{INTRODUCCIÓN}

La educación ambiental en el mundo se percibe como un tema poco abordado por el sistema educativo, los indicadores de medición se enfocaron en el tratamiento del uso de la basura y de materiales no biodegradables que genera la propia naturaleza (Alvarez, 2016). A nivel mundial, el tema ambiental es preocupante; un informe realizado por Unicef (2019) observó el impacto negativo sobre los efectos del calentamiento global, a raíz de la acumulación excesiva de focos contaminantes generados en ciudades desarrolladas, teniendo como causales la industrialización donde no se vienen aplicando indicadores de control para cuidar el medio ambiente.

El problema del reciclaje en América Latina se incrementa considerablemente (Thefoodtech.com, 2021), una tercera parte de los desechos terminan en basurales que no tienen ningún tipo de control y contaminan el ambiente; es en estos lugares donde la población se encuentra expuesta a enfermedades; un factor que perturba la salud de la población y vienen contaminando los suelos, el agua y el aire, estos sucesos son una muestra de la falta de concientización del problema y no se viene abordando desde la etapa preescolar en temas que generen cultura ambiental. (ONU, 2018, párr. 2). A pesar que las instituciones educativas cuentan con cursos relacionados al medio ambiente, todavía falta trabajar mucho más el cuidado y su preservación; es notable que el tema pasa por la carencia de estrategias de tipo educativo para poder darle una mayor sostenibilidad a la gestión de programas como por ejemplo el de reciclaje y de esta manera ir formando generaciones que crezcan con un aprendizaje continuo y valoren la importancia que tiene el medio ambiente para poder vivir de manera sana.

En el caso de Perú, un país con ciudades extensas como Arequipa, Trujillo, Piura, entre otras que albergan una amplia cantidad de población, y se presencia cómo la acumulación excesiva de basura y residuos en los principales distritos evidencian un problema ambiental por la contaminación que este produce. En el ámbito educativo, en el Currículo Nacional de la Educación Básica (CNEB) establece al enfoque ambiental como proceso formativo que orienta en la idoneidad de ciudadanos con formación y conciencia crítica y colectiva acerca del problema ambiental, expresa que los niños deben tener una educación activa, que no sólo sea la de innovar cultura y educación (MINEDU, 2016), sino que les conceda tener un cambio sobre la visión en la mejora de las prácticas de enseñanza - aprendizaje, sensibilizando a la comunidad educativa sobre el compromiso 
de trabajar diariamente para mejorar el medio ambiente, reforzándose a través de iniciativas educativas una nueva manera de fomentar la cultura del reciclaje.

La investigación determinó en qué medida un programa de reciclaje mejora la expresión plástica en niños de cuatro años de educación inicial de la IE N 1678 Rayitos de Luz del distrito de Nuevo Chimbote, considerando que es necesario formar a los estudiantes en el cuidado del medio ambiente mediante el impulso de las prácticas de reciclaje y desarrollar sus habilidades creativas a partir de la expresión plástica. Por lo tanto, existió un aspecto sustancial enfocado en un tema de realidad social, que en el manejo diario y en la convivencia con los alumnos ha sido notoria la poca difusión de temas vinculados con el aspecto ambiental.

Respecto a la problemática, la educación ambiental, es poco abordada en las escuelas, así como; el tratamiento de la basura y el uso de materiales no biodegradables, la creación de nuevos productos, la contaminación que estos genera, originándose el aumento de residuos sólidos desmedidamente, sin pensar en disminuir o en el daño que crean en el planeta (Pablo, 2019, p. 14); es así que, a pesar de plantear estrategias o propuestas de diferentes políticas de conservación del medio ambiente que se ponen en práctica, en diversos países, no se vienen logrando los resultados esperados. Asimismo, con esta investigación se buscó fomentar en los estudiantes, el respeto por su entorno, el medio al que se pertenece, proponiendo actividades para las docentes en activar dentro de sus experiencias de aprendizajes, temas que generen conciencia ambiental, desarrollando su creatividad e imaginación, para elaborar manualidades con recursos poco usables y convertirlos en nuevos productos útiles para la sociedad. De acuerdo a los hechos, causas y consecuencias del problema se formuló la siguiente interrogante ¿En qué medida el Programa de Reciclaje mejoró la expresión plástica en niños de 04 años de la IE $\mathrm{N}^{\circ} 1678$ "Rayitos de Luz" - 2021?.

El estudio se justifica en los siguientes aspectos: conveniencia, porque los problemas del medio ambiente se analizaron a una temprana edad. Por el lado de la relevancia social, el estudio se orientó en dotar a los profesores y estudiantes, herramientas que oriente sus acciones del diario vivir para fomentar en los niños y niñas sus actividades de expresión plástica, así como su creatividad y cuidado del medio que les rodea. En la implicancia práctica, el uso de insumos comunes que son desechados deben ser puestos en buen recaudo y no contaminar. Por otro lado, el valor teórico del proyecto se fundamentó en 
los aportes teóricos relacionados con el reciclaje y la expresión plástica. La justificación metodológica, permitió emplear de manera recreativa diferentes formas de aprender, reutilizando y reciclando, así también para futuras investigaciones.

El objetivo de estudio ha sido demostrar que la aplicación del Programa de Reciclaje mejora la expresión plástica en niños de 04 años de edad en la I.E. $N^{\circ} 1678$ - 2021. Se plantearon las hipótesis que se comprobaron mediante un modelo matemático: $\mathrm{H}_{1} \mathrm{La}$ aplicación del Programa de Reciclaje mejoraría significativamente la expresión plástica en niños de 04 años en la I.E. $\mathrm{N}^{\circ} 1678$ - 2021; y $\mathrm{H}_{0}$ la aplicación del Programa de Reciclaje no mejoraría significativamente la expresión plástica en niños de 4 años en la I.E N ${ }^{\circ} 1678-2021$.

En cuanto a las variables y dimensiones, conceptualizando el currículo, la educación inicial se convierte en la primera etapa de la EBR, la cual se enfoca en la atención a los niños que tienen menos de 6 años, constituyendo un contexto trascendental de aprendizaje en el infante, pues en ella se programan las bases para desarrollar el potencial biológico, de afecto, conocimiento y social de las personas, esencialmente, sirve de base para la formación de las personas (Yager, 2018).

Analizando la expresión plástica, es de especial importancia para el niño porque a través de sus representaciones, trazos, dibujo y construcciones se puede recolectar información sobre su comportamiento social en casa, además que la expresión plástica se desarrolla en el niño su imaginación, creatividad, su infancia, el animismo y la motricidad fina, es necesario mencionar que también la educación plástica ayuda al niño a dominar técnicas plásticas, desarrollar su capacidad de observación visual, auditiva, olfativa y táctil; en general la percepción sensorial y el origen de la estética. El programa estuvo evaluado permanentemente a través de una lista de cotejo que fue operacionalizada, donde se concluyó que el hábitat estuvo conformado por elementos bióticos y abióticos, así como también por entender valores sociales, naturales y culturales, influyentes en la vida emocional del hombre.

Castells (2012) detalló al reciclaje como la operación complicada, en donde permite todo un proceso que se debe realizar para la elaboración de un nuevo material a partir de residuos. Por otro lado, la pregunta con qué factores se relacionan los comportamientos de los individuos con respecto al reciclaje después de comprender la importancia del reciclaje a nivel internacional ha pasado a primer plano tanto psicológica como 
sociológicamente en términos tanto de la educación ambiental como del reciclaje (Allen, Davids, \& Soskin, 2017).

Describiendo las dimensiones de la expresión plástica: Para operacionalizar la variable expresión plástica, se consideraron los siguientes dominios: dominio conceptual, los contenidos se transforman en aprendizajes, partiendo de los conocimientos previos de cada infante, que a su vez se interrelacionan con otros contenidos (Cassassus, 2018). Se tomó en cuenta que, no basta con obtener información y tener conocimientos teóricos de áreas determinadas ya sea científica o cotidiana, sino entenderlos. (Solé, 2018).

Dominio procedimental, es el desarrollo de una serie de actividades que promueven el logro de un fin, y este se debe ver reflejado en el rendimiento. El estudiante es el actor principal que realiza los procesos en los contenidos (Bedoya y Guerrero, 2018).

Dominio actitudinal, en esta dimensión el estudiante demuestra la aplicación de los valores, creencias, reglas de conducta, capacidad, habilidades y competencias reflejadas en las actitudes que conducen al equilibrio personal y a la buena convivencia. (Allen y Seaman, 2019). Se concluye que los tres tipos de dominios citados, cuentan con el mismo nivel de interés y se debe abordar de forma integrada por el docente (Matos, 2015).

La principal teoría que fundamenta el estudio es la pedagogía infantil, debido al tratamiento de las variables, estas se anclan también a la psicología cognitiva, antropología educativa y paradigmas educativos de la primera infancia en cuanto a la aplicación de un programa o plan educativo que permitiría mejorar y potenciar los conocimientos en materia social del cuidado del medio ambiente. Un estudio presentado por la Organización Educativa Waece.Org (2021) señaló que la pedagogía infantil tiene entre sus puntos neurálgicos potenciar la construcción social de temas que se deben manejar desde la educación inicial.

\section{ESTRATEGIAS METODOLÓGICAS O MATERIALES Y MÉTODOS}

La investigación pertenece al enfoque cuantitativo, que de acuerdo con Hernández et al (2014) “emplea el razonamiento lógico o el deductivo, asimismo tiene las siguientes características: de enfoque cuantitativo, específico y delimitado" (p. 51). El estudio es de tipo descriptivo - experimental. El diseño de estudio correspondió al pre experimental, ya que el trabajo se realizó un análisis deliberado de las variables, y los fenómenos fueron analizados en el pre test (prueba de entrada) en su entorno natural. Posteriormente se aplicó una segunda prueba para poder medir el efecto del Plan que se propone como un 
pos test (prueba de salida). La característica principal se especifica en un solo grupo Pre y Post Test, tal como se aprecia en el siguiente esquema.

$$
\mathbf{G}_{\mathbf{e}}=\mathbf{O}_{1}
$$

\section{Donde:}

$\mathrm{G}_{\mathrm{e}}=$ grupo Experimental.

$\mathrm{O}_{1}=$ grupo Pre y Post Test

$\mathrm{O}_{2}=$ Pre y Post Test.

$\mathrm{X}=$ Variable Independiente.

$\mathrm{P}=$ Propuesta.

La población objeto de estudio del presente trabajo estuvo comprendido por 189 infantes de 3, 4 y 5 años en la IE Rayitos de Luz, ubicado en el distrito Nuevo Chimbote. Siendo un muestreo de tipo censal tomándose la totalidad. La técnica empleada para la recolección de datos ha sido la Guía de Observación. La validación del instrumento se realizó a través de la medición estadística mediante el Alfa de Cronbach, que arrojó un nivel de confianza de 0.804. El método de análisis de datos empleados se realizó a través de la utilización de tablas de frecuencia, también de gráficos y estadísticas para la validación de la hipótesis; para el caso, se aplicó la distribución de frecuencias, la media aritmética y la prueba de "medias" mediante la prueba " $T$ " de Student para muestras relacionadas, lo que permitió realizar la comparación de las medias de dos series de mediciones en los mismos estadísticos utilizados.

\section{RESULTADOS Y DISCUSIÓN}

En el siguiente acápite se evidencia el resultado de las variables y dimensiones de estudio, los cuales parte de la ficha de observación y sesiones de clase aplicadas.

Tabla 1: Resultado del pre y post en la Expresión Plástica 


\begin{tabular}{lcccc}
\hline & \multicolumn{2}{c}{ Pre Test } & \multicolumn{2}{c}{ Post Test } \\
\hline Nivel & fi & \% & fi & \% \\
\hline Malo & 19 & $63,3 \%$ & 0 & $0,0 \%$ \\
Regular & 8 & $26,7 \%$ & 2 & $6,7 \%$ \\
Bueno & 3 & $10 \%$ & 5 & $16,7 \%$ \\
Muy Bueno & 0 & $0,0 \%$ & 23 & $76,6 \%$ \\
\hline Total & $\mathbf{3 0}$ & $\mathbf{1 0 0 \%}$ & $\mathbf{3 0}$ & $\mathbf{1 0 0 , 0 \%}$ \\
\hline
\end{tabular}

Fuente. Base de datos del Pre y Post Test aplicado a estudiantes de 04 años en la IEI

\section{Interpretación:}

En la Tabla 1 se aprecia que de una muestra de 30 estudiantes en el pre test el 63.3\% (19 alumnos) se encontraban en un nivel malo, el 26.7\% (8 estudiantes) rindieron en un nivel regular, el 10\% (3 alumnos) tuvieron una calificación en un nivel bueno. Después de aplicar el programa los alumnos mejoran de manera significativa, el 76.6\% rindieron de manera muy buena, el $16.7 \%$ pasaron a tener una calificación buena, y el $6.7 \%$ obtuvieron una calificación regular.

Tabla 2: Resultado del pre y post test en la dimensión Dominio Conceptual para mejorar la Expresión Plástica

\begin{tabular}{lcccc}
\hline & & Pre Test & \multicolumn{2}{c}{ Post Test } \\
\hline Nivel & fi & \% & fi & \% \\
\hline Malo & 24 & $80 \%$ & 0 & $0,0 \%$ \\
Regular & 6 & $20 \%$ & 4 & $13,3 \%$ \\
Bueno & 0 & $0,0 \%$ & 4 & $13,3 \%$ \\
Muy Bueno & 0 & $0,0 \%$ & 22 & $73,4 \%$ \\
\hline Total & $\mathbf{3 0}$ & $\mathbf{1 0 0 \%}$ & $\mathbf{3 0}$ & $\mathbf{1 0 0 , 0 \%}$ \\
\hline
\end{tabular}

Fuente. Base de datos del Pre y Post Test aplicado a estudiantes de 04 años en la IEI

\section{Interpretación:}

En la Tabla 2 referida a la dimensión dominio conceptual, en el pre test el 80\% (24 alumnos) se encontraban en un nivel malo, el 20\% (6 estudiantes) rindieron en un nivel regular. Sin embargo, después de aplicar el programa "Programa de Reciclaje para mejorar la Expresión Plástica" los alumnos mejoran de manera significativa en el dominio conceptual, el 73.4\% (22 alumnos) rindieron de manera muy buena, el 13.3\% (4 alumnos) pasaron a tener una calificación buena, el 13.3\% (4 alumnos) obtuvieron una calificación regular. 
Tabla 3: Resultado del pre y post test en la dimensión Dominio Procedimental para mejorar la Expresión Plástica

\begin{tabular}{lcccc}
\hline \multirow{2}{*}{ Nivel } & \multicolumn{2}{c}{ Pre Test } & \multicolumn{2}{c}{ Post Test } \\
\cline { 2 - 5 } & $\mathbf{f i}$ & $\mathbf{\%}$ & $\mathbf{f i}$ & $\mathbf{\%}$ \\
Malo & 20 & $66,7 \%$ & 0 & $0,0 \%$ \\
Regular & 10 & $33,3 \%$ & 3 & $10,0 \%$ \\
Bueno & 0 & $0,0 \%$ & 4 & $13,3 \%$ \\
Muy Bueno & 0 & $0,0 \%$ & 23 & $76,7 \%$ \\
\hline \multicolumn{1}{c}{ Total } & $\mathbf{3 0}$ & $\mathbf{1 0 0 \%}$ & $\mathbf{3 0}$ & $\mathbf{1 0 0 , 0 \%}$ \\
\hline
\end{tabular}

Fuente Base de datos del Pre y Post Test aplicado a estudiantes de 04 años en la IEI

\section{Interpretación:}

En la Tabla 3 referida al resultado de la dimensión Dominio Procedimental, en el pre test el 66.7\% (20 alumnos) se encontraban en un nivel malo, el 33.3\% (10 estudiantes) rindieron en un nivel regular. Sin embargo, después de aplicar el programa "Programa de Reciclaje para mejorar la Expresión Plástica" los alumnos mejoran de manera significativa, el 76.7\% (23 alumnos) rindieron de manera muy buena, el 13.3\% (4 alumnos) pasaron a tener una calificación buena, el 10\% (3 alumnos) obtuvieron una calificación regular.

Tabla 4: Resultado del pre y post test en la dimensión Dominio Actitudinal para mejorar la Expresión Plástica

\begin{tabular}{lcccc}
\hline \multirow{2}{*}{ Nivel } & \multicolumn{2}{c}{ Pre Test } & \multicolumn{2}{c}{ Post Test } \\
\cline { 2 - 5 } & $\mathbf{f i}$ & $\mathbf{\%}$ & $\mathbf{f i}$ & $\mathbf{\%}$ \\
\hline Malo & 24 & $80 \%$ & 0 & $0,0 \%$ \\
Regular & 6 & $20 \%$ & 4 & $13,3 \%$ \\
Bueno & 0 & $0,0 \%$ & 4 & $10 \%$ \\
Muy Bueno & 0 & $0,0 \%$ & 23 & $76,7 \%$ \\
\hline \multicolumn{1}{c}{ Total } & $\mathbf{3 0}$ & $\mathbf{1 0 0 \%}$ & $\mathbf{3 0}$ & $\mathbf{1 0 0 , 0 \%}$ \\
\hline
\end{tabular}

Fuente Base de datos del Pre y Post Test aplicado a estudiantes de 04 años en la IEI

\section{Interpretación:}

En la Tabla 4 referida a la dimensión dominio actitudinal, se aprecia que en el pre test el $80 \%$ se encontraban en un nivel malo, el $20 \%$ rindieron en un nivel regular. Sin embargo, después de aplicar el programa los alumnos mejoran de manera significativa en la dimensión dominio actitudinal, el 76.7\% rindieron de manera muy buena, el 10\% pasaron a tener una calificación buena, el $13.3 \%$ obtuvieron una calificación regular. 
En relación al análisis inferencial, en la Tabla 5 se muestran los resultados de la prueba de hipótesis general.

Tabla 5: Resultados de Prueba de T Student para la variable Expresión Plástica

\begin{tabular}{cccccc}
\hline & Media & N & DE & Err.Est.Media \\
\hline Pareja 1 & Pre Test & 33.4 & 30 & 7.32 & 1.34 \\
\hline & Pos Test & 65.67 & 30 & 8.4 & 1.53 \\
\hline
\end{tabular}

Fuente: Base de datos procesada en SPSS (2021)

\section{Interpretación:}

Analizando la prueba de T Student en la Tabla 5 se muestra la existencia de diferencias significativas entre la prueba tomada en el pre test y el pos test, estableciéndose una media de 32.27 puntos con una DE en 10.43. Se concluye que la aplicación del Programa de Reciclaje mejora la Expresión Plástica, aceptándose la hipótesis alterna y se rechaza la hipótesis nula.

Analizando los resultados de la Hipótesis Especifica 1, acerca de la aplicación del Programa de Reciclaje mejora el dominio conceptual de la Expresión Plástica en niños de 04 años en la I.E. $N^{\circ} 1678$ - 2021. En la Tabla 6 se muestran los resultados de la prueba T Student, donde se aprecia que los alumnos en el Pre Test obtuvieron un puntaje de acuerdo a la media de 8.73 cuya situación en calificación estuvo en una escala denominada Mala (rango de 05 - 09); sin embargo, al aplicar el Programa para mejorar la Expresión Plástica los alumnos en el Pos Test mejoran de manera considerable el domino conceptual logrando una calificación de 17.07 en una escala muy buena.

Tabla 6: Resultados de Prueba de T Student para la dimensión Dominio Conceptual

\begin{tabular}{llcccc}
\hline & & Media & N & DE & Err.Est.Media \\
\hline Pareja 1 & Pre Test & 8.73 & 30 & 1.2 & 0.22 \\
& Pos Test & 17.07 & 30 & 2.39 & 0.44 \\
\hline
\end{tabular}

Fuente: Base de datos procesada en SPSS (2021)

\section{Interpretación:}

Analizando la prueba de T Student en la Tabla 6 existen diferencias significativas entre la prueba tomada en ambos test, estableciéndose una media de 8.33 puntos con una DE en 2.45. De acuerdo al planteamiento de hipótesis se deduce que al aplicar el Programa de Reciclaje mejora el dominio conceptual de la Expresión Plástica. 
Por otro lado, en los resultados de la Hipótesis Especifica 2. Al aplicar el Programa de Reciclaje mejora de manera positiva el dominio procedimental de la Expresión Plástica en niños de 04 años en la I.E. $N^{\circ} 1678$ - 2021. En la Tabla 7 se muestran los resultados de la prueba T Student, donde se aprecia que los alumnos en el Pre Test obtuvieron un puntaje de acuerdo a la media de 18.07 cuya situación en calificación estuvo en una escala denominada Mala (rango de 10-18); sin embargo, al aplicar el Programa para mejorar la Expresión Plástica los alumnos en el Pos Test mejoran de manera considerable el domino procedimental logrando una calificación de 34.73 en una escala buena.

Tabla 7: Resultados de Prueba de T Student para la Dominio Procedimental

\begin{tabular}{llcccc}
\hline & & Media & N & DE & Err.Est.Media \\
\hline Pareja 1 & Pre Test & 18.07 & 30 & 4.76 & 0.87 \\
& Pos Test & 34.73 & 30 & 4.36 & 0.8
\end{tabular}

Fuente: Base de datos procesada en SPSS (2021)

\section{Interpretación:}

Analizando la prueba de T Student en la Tabla 7 se muestra que existen diferencias significativas entre la prueba tomada en el pre test y el pos test, estableciéndose una media de 16.67 puntos con una DE en 6.41. De acuerdo al planteamiento de hipótesis se deduce que la aplicación del Programa de Reciclaje mejora el dominio procedimental.

Finalmente, en los resultados de la Hipótesis Especifica 3. La aplicación del Programa de Reciclaje mejora significativamente el dominio actitudinal de la Expresión Plástica en niños de 04 años en la I.E. $N^{\circ} 1678$ - 2021. En la Tabla 8 se muestran los resultados de la prueba T Student, donde se aprecia que los alumnos en el Pre Test obtuvieron un puntaje de acuerdo a la media de 6.6 cuya situación en calificación estuvo en una escala denominada Mala (rango de 04-07); sin embargo, al aplicar el Programa para mejorar la Expresión Plástica los alumnos en el Pos Test mejoran de manera considerable el domino actitudinal logrando una calificación de 13.87 en una escala buena.

Tabla 8: Resultados de Prueba de T Student para la Dominio Actitudinal

\begin{tabular}{cccccc}
\hline & Media & N & DE & Err.Est.Media \\
\hline Pareja 1 & Pre Test & 6.6 & 30 & 2.39 & 0.44 \\
& Pos Test & 13.87 & 30 & 2.13 & 0.39 \\
\hline
\end{tabular}

Fuente: Base de datos procesada en SPSS (2021)

\section{Interpretación:}


Analizando la prueba de T Student en la Tabla 8 se muestra que existen diferencias significativas entre la prueba tomada en el pre test y el pos test, estableciéndose una media de 7.27 puntos con una DE en 3.17. De acuerdo al planteamiento de hipótesis se deduce que la aplicación del Programa mejora el dominio actitudinal de la Expresión Plástica.

\section{DISCUSIÓN}

En relación con los resultados de la Hipótesis General (HG) se comprobó que la aplicación del programa mejora significativamente la expresión plástica en la muestra tomada de estudiantes de las IEI. Los resultados fueron congruentes con el estudio de Apaza y Calisaya (2019) quienes concluyeron que el reciclaje como actividad de desarrollo y aplicación en el aula es eficaz y permiten de manera paulatina que se fortalezcan la capacidad y habilidad de los infantes en la IEI, en cuanto al análisis de los tesistas referentes el 68\% se ubican en nivel de logro previsto en 19 infantes representados por el 76\%. También, la investigación de Huamán (2019) logró demostrar resultados muy similares al hallazgo de la HG, estadísticamente se obtuvo un T Student mayor que la $\mathrm{T}$ teórica $(22,24<1,98)$. Asimismo, el estudio de Castellano, De Carolis, D’Errico, Macchiarulo, y Rossano (2021), evidenciaron mejoras significativas en el aprendizaje del reciclaje a través de la aplicación del experimento, estadísticamente se obtuvo un nivel alto de $95 \%$ en el estadístico de prueba T Student.

En cuanto a la HE 1, donde se comprobó que al aplicar el programa propuesto se logran mejorar del dominio conceptual de la expresión plástica. Los resultados demostraron que al analizar la prueba de T Student se estableció la existencia de diferencias significativas de la prueba tomada en el pre test y el post test, estableciéndose una media de 8.33 puntos con una DE en 2.45. Los resultados son congruentes con el estudio realizado por Bedoya y Guerrero (2018) quienes evidenciaron que a un nivel de confianza (NC) del 95\% la aplicación de programas de dominio conceptual en el uso del material reciclado influyó de manera favorable en la coordinación viso-manual en los alumnos de 05 años. De la misma manera el estudio de Flores y Limache (2018) al aplicarse la prueba estadística obtuvieron un resultado que la $\mathrm{T}$ es (9.980) y la $\mathrm{T}$ tabulada (1.7346) siendo una diferencia de (8.2454), evidenciándose que el programa de reciclado es significativo en el cuidado del entorno natural que muestran los alumnos.

En cuanto a la HE 2, se comprobó que al aplicar el programa se establecieron mejoras en el dominio procedimental de la expresión plástica en los infantes de la I.E.I. Los 
resultados demostraron que al aplicar la T Student se obtuvieron diferencias favorables entre la prueba tomada en el pre test y el pos test, estableciéndose una media de 16.67 puntos con una DE en 6.41. Los resultados son congruentes con el estudio realizado por De Los Ríos, G. (2018), la prueba estadística señala que los alumnos mejoran el nivel de concientización ambiental en un 95\%, de esta forma se demuestra que el dominio procedimental es un eje importante en el desarrollo de este plan.

En cuanto a la HE 3, se comprobó que la aplicación del programa mejora significativamente el dominio actitudinal de la expresión plástica en los niños de 04 años. Los resultados demostraron que al analizar la prueba de T Student se muestra que existen diferencias favorables estadísticamente entre la prueba tomada en el pre test y el pos test, estableciéndose una media de 7.27 puntos con una DE en 3.17. en los contenidos actitudinales, que constituyen valores, normas, creencias y actitudes que conducen al equilibrio personal y a la convivencia social. (Seaman, 2019). Se destaca tres dominios característicos de amplio interés para el aprendizaje de los infantes y se debe abordar en la actividad de las docentes de manera integral (Matos, 2015).

En conclusión, la expresión plástica es una herramienta de uso del estudiante para que se exprese en los distintos lenguajes. Así como la expresión plástica, es una forma de representar y comunicar, empleando un lenguaje que sea expresado con la manipulación de los plásticos y de técnicas que van a dar lugar al proceso creador. (Cruz y otros, 2015. p. 24-25). De acuerdo a López (2009) manifestaron que la expresión es necesaria en el infante que le permitió, primeramente, adaptarse al mundo y en un segundo momento desarrollarse como un ser creativo, imaginativo y autónomo.

\section{CONCLUSIÓN O CONSIDERACIONES FINALES}

La aplicación del programa de reciclaje mejora significativamente la expresión plástica en los niños de 04 años que participaron. Se demostró diferencias significativas favorables en el análisis de la prueba de T Student, tanto en la prueba del pre test y el pos test, estableciéndose una media de 32.27 puntos con una DE en 10.43. De acuerdo al planteamiento de hipótesis la aplicación del programa mejora la expresión plástica en niños de 04 años en la institución descrita.

También al aplicar el programa de reciclaje se lograron mejoras en el dominio conceptual de la expresión plástica. Los resultados mediante la prueba de T Student establecen la existencia de diferencias favorables entre la prueba tomada en el pre test y el pos test, 
estableciéndose una media de 8.33 puntos con una DE en 2.45. En tal sentido, al usar el material reciclado, se ha permitido de manera efectiva que los niños y niñas de la IEI logren un desarrollo positivo en el dominio conceptual.

El programa de reciclaje mejora el dominio procedimental de la expresión plástica, los resultados demostraron que al analizar la prueba de T Student se establecen diferencias significativas entre la prueba tomada en el pre test y el pos test, estableciéndose una media de 16.67 puntos con una DE en 6.41. Por tanto, el uso del material reciclado, permitió que los niños y niñas logren mejorar la coordinación de dominio procedimental.

Finalmente, la aplicación del programa de reciclaje mejora significativamente el dominio actitudinal de la expresión plástica. Demostrándose que al analizar la prueba de T Student se muestran diferencias significativas entre la prueba tomada en el pre test y el pos test, estableciéndose una media de 7.27 puntos con una DE en 3.17. La puesta en práctica del material reciclado por parte de las docentes de educación inicial ha permitido que los niños de la IEI logren desarrollar de manera positiva la coordinación de dominio actitudinal.

\section{LISTA DE REFERENCIAS}

Allen, I., \& Seaman, J. (2018). Online nation: Five years of growth in online learning. New York: Newburyport, MA: The Sloan Consortium. Retrieved from.

Allen, I., \& Seaman, J. (2019). Learning on demand online education in the United States.

New York: Newburyport, MA: The Sloan Consortium. Retrieved from.

Álvarez, C. (2016). Reciclaje y su aporte en la educación ambiental. Tesis presentada a Coordinación de Facultad de Humanidades, Universidad Rafael Landívar, Quetzaltenango. Tesis Doctoral con Orientación en Administración y Evaluación Educativas. Recuperado de http://biblio3.url.edu.gt/Tesario/2013/05/09/AlvarezCarina.pdf

Apaza, G., \& Calisaya, V. (2019). El 1 reciclaje como actividad para la conservación del medio ambiente en los niños y niñas de 4 años A. Juliaca: Universidad del Altiplano.

Bedoya, J., \& Guerrero, R. (2018). Material reciclado en la coordinación viso-manual en los estudiantes de 05 años de la I.E.I “Casa De Los Traviesos"- Huancavelica. Huancavelica: Universidad Nacional De Huancavelica. 
Castellano, G., De Carolis, B., D’Errico, F., Macchiarulo, N., \& Rossano, V. (2021). PeppeRecycle: Improving Children's Attitude Toward Recycling by Playing with a Social Robot. International Journal of Social Robotics, 12(1), 12.

Cassassus, J. (2018). Problemas de la gestión educativa en América Latina. Congreso Nacional Redue.

Castells, E. (2012). Reciclaje de residuos industriales. 2ª ed. España. Edit. Díaz de Santos. De Los Ríos, G. (2018). Aplicación del plan nacional de educación ambiental en el desarrollo de la conciencia ambiental de los estudiantes de primaria en las escuelas ecoeficientes del distrito de San Juan de Lurigancho UGEL 05. Lima: UIGV. Tesis de Doctor en Educación. Encontrado en https://renati.sunedu.gob.pe/handle/sunedu/2915792.

Flores, N., y Limache, B. (2018). Material reciclado en el cuidado del entorno natural con los niños y niñas de 5 años en la Institución Educativa Inicial Divino Niño Jesús - Villa Del Lago - Puno 2018. Tesis doctoral, Universidad Nacional Del Altiplano, Puno.

Hernández, R., Fernández, C., \& Baptista, P. (2014). Metodología de la Investigación. México: McGraw-Hill.

Huamán, V. (2019) Manejo de residuos sólidos en la Universidad Nacional del centro. Huancayo: UNC

Matos, H. (2015). Nueva Cultura Evaluativa. Lima: Servicio Gráficos Silva.

Ministerio de Educación (31 de Julio de 2016). Currículo Nacional de la Educación Básica. Recuperado de http://www.minedu.gob.pe/curriculo/pdf/curriculonacional-2016.pdf

Naciones Unidas. (12 de octubre de 2018). Cómo la basura afecta al desarrollo de América Latina: Objetivos de Desarrollo Sostenible. Recuperado de https://news.un.org/es/story/2018/10/1443562

Organización Educativa Waece.Org. (2021). Paradigmas y principios de la educación infantil o inicial. Waece.org. Recuperado de http://www.waece.org/forma_docente/informe/capitulo2_4.php

Pablo, F. (31 de Julio de 2019). Diseño Curricular Básico Nacional. Recuperado de http://www.iesppmfgb.edu.pe/wp-content/uploads/2020/02/Dise\%C3\%B1o- 
Curricular-B\%C3\%A1sico-Nacional-2019-Educaci\%C3\%B3n-Primaria09082019.pdf

Solé, I. (2018). Estrategias de lectur. Barcelona, España: Editorial Graó.

Thefoodtech.com. (11 de Enero de 2021). El problema del Reciclaje en América Latina. Recuperado de https://thefoodtech.com/historico/el-problema-del-reciclaje-enamerica-latina/

UNICEF. (31 de diciembre de 2019). Medio ambiente y cambio climático. El cambio climático y la degradación del medio ambiente socavan los derechos de todos los niños. Recuperado de https://www.unicef.org/es/medio-ambiente-cambioclimatico

Yager, R. (2018). Achieving the staff development model advocated in the national standards. New York: Standards. Science Educator. 